\title{
Cooking frequency may enhance survival in Taiwanese elderly
}

\author{
Rosalind Chia-Yu Chen ${ }^{1}$, Meei-Shyuan Lee ${ }^{2,3}$, Yu-Hung Chang ${ }^{4}$ and \\ Mark L Wahlquist ${ }^{1,2,3, *}$ \\ 'Division of Preventive Medicine and Health Services Research, Institute of Population Health Sciences, National \\ Health Research Institutes, 35 Keyan Road, Zhunan, Miaoli County 350, Taiwan, Republic of China: ${ }^{2}$ School of \\ Public Health, National Defense Medical Center, Taipei, Taiwan, Republic of China: ${ }^{3}$ Asia Pacific Health and \\ Nutrition Centre, Monash Asia Institute, Monash University, Melbourne, Australia: ${ }^{4}$ Division of Health Policy and \\ Translation, Institute of Population Health Sciences, National Health Research Institutes, Zhunan, Taiwan, \\ Republic of China
}

Submitted 4 October 2011: Final revision received 20 March 2012: Accepted 30 March 2012: First published online 11 May 2012

\begin{abstract}
Objective: To investigate the association between cooking behaviour and longterm survival among elderly Taiwanese.

Design: Cohort study. The duration of follow-up was the interval between the date of interview and the date of death or 31 December 2008, when censored for survivors. Information used included demographics, socio-economic status, health behaviours, cooking frequencies, physical function, cognitive function, nutrition knowledge awareness, eating out habits and food and nutrient intakes. These data were linked to death records. Cox proportional-hazards models were used to evaluate cooking frequency on death from 1999 to 2008 with related covariate adjustments.

Setting: Elderly Nutrition and Health Survey in Taiwan, 1999-2000.

Subjects: Nationally representative free-living elderly people aged $\geq 65$ years ( $n$ 1888).

Results: During a 10-year follow-up, 695 participants died. Those who cooked most frequently were younger, women, unmarried, less educated, non-drinkers of alcohol, non-smokers, without chewing difficulty, had spouse as dinner companion, normal cognition, who walked or shopped more than twice weekly, who ate less meat and more vegetables. Highly frequent cooking $(>5$ times/week, compared with never) predicted survival (hazard ratio $(\mathrm{HR})=0 \cdot 47 ; 95 \% \mathrm{CI}, 0 \cdot 36,0 \cdot 61$ ); with adjustment for physical function, cognitive function, nutrition knowledge awareness and other covariates, HR was 0.59 (95\% CI, 0•41, 0.86). Women benefited more from cooking more frequently than did men, with decreased HR, $51 \% v .24 \%$, when most was compared with least. A 2-year delay in the assessment of survivorship led to similar findings.

Conclusions: Cooking behaviour favourably predicts survivorship. Highly frequent cooking may favour women more than men.
\end{abstract}

The concept of 'successful ageing' gained currency in the mid-20th century and was progressively promoted ${ }^{(1)}$. One of its three components was defined as 'active engagement with life'. 'Healthy ageing' was conceptualized with more concern for the quality of life vital for development, and achievable when 'appropriate nutrition and healthy eating at an early age' were ensured according to $\mathrm{WHO}^{(2,3)}$. This underscored the importance of food habits in healthseeking behaviour for ageing people. Such habits are likely to influence both disability and mortality outcomes. Food habits may or may not extend to food preparation and whether they do may be relevant to health outcomes, making food preparation a health-promoting behaviour ${ }^{(4)}$.
Cooking, in the broad sense, refers to food preparation which provides an opportunity for personal involvement in the food system. Cooking requires the selection, measurement and combining of ingredients in an ordered procedure to achieve the desired result. Therefore, it is an activity which requires physical and mental health. There is a relationship between food preparation and cooking skills and food choice within a family ${ }^{(5)}$. In the community and household, the kitchen is a key social location, a situation referred to as 'commensality'(6).

Life stage has a direct impact on cooking behaviours and attitudes ${ }^{(7)}$. Cooking behaviour varies with age. Younger people may look to cook more creatively while 
seniors balance convenience and comfort in their cooking. Ethnicity influences mainstream cooking culture ${ }^{(8)}$. In addition, cooking has a pronounced gender variation ${ }^{(9)}$. Women are still probably the primary role models and teachers of cooking and food preparation skills across age and socio-economic groups, as illustrated by notable examples of such leadership, although men's contribution as cooking mentors, other than as doyen chefs, would appear to be on the increase ${ }^{(10)}$. Taste, nutritional value, cost and time for preparation are the primary and often conflicting factors which influence food choice and preparation decisions ${ }^{(5)}$.

Elders may be less able or willing to be participants in a number of food consumption trends. Increasingly food is eaten away from home for convenience and tends to be lower in nutritional quality than food prepared at home, although healthy alternatives may still be desired. More affluent consumers may seek a variety of foods and cuisines along with convenience, entertainment and enjoyable dining experiences ${ }^{(11)}$. For more home-bound elders, there must be questions about the health consequences of the aggregate of limited shopping ${ }^{(12)}$, restraints on eating out and whether and how often it is possible to cook at home with or without help.

Cooking has been an important part of social life and household economics, especially for older generations. We have investigated whether cooking activity can contribute to survivorship, independent of physical and cognitive function, by linking study data to the National Death Registry for decedents' time of death.

\section{Methods}

\section{Study population}

The present study used data from the Elderly Nutrition and Health Survey in Taiwan (NAHSIT Elderly), a nationally representative sample of the free-living elderly aged $\geq 65$ years, conducted in 1999 and $2000^{(13)}$. A total of 1937 participants completed the household interview and 1900 had a verifiable national identity number which would allow linkage to the National Death Registry. Seven, two and three participants had no cooking information, unreasonable response about their cooking status or an incorrect date of death, respectively. This left 1888 eligible participants for analysis. All participants signed informed consent. The ethics committees of the National Health Research Institutes and Academia Sinica in Taiwan approved the study protocol.

\section{Measurements}

\section{Cooking frequency}

Cooking frequency was measured by the following question: 'Do you have to cook or prepare food for yourself or help with these? Do not include ready-to-eat food'. The response options were 'never', '1-2 times a week', '3-5 times a week' and 'more than 5 times a week'. Whether help was provided by a food preparer was asked separately, and the two responses were integrated as required in the models developed. In particular, the following question was asked: 'If you prepare three meals a day for yourself, who is usually responsible in buying the ingredients for cooking?', with possible answers of 'never prepare food for myself', 'self', 'children or relatives', 'friends or neighbours', 'housemaid' and 'others (please indicate)'.

\section{Dietary intake}

The dietary information was collected by a $24 \mathrm{~h}$ dietary recall at interview. The mean daily food intakes per $4184 \mathrm{~kJ}(1000 \mathrm{kcal})$, as well as mean daily energy intake and nutrient densities per $4184 \mathrm{~kJ}$ ( $1000 \mathrm{kcal})$ for selected nutrients, were calculated.

\section{Covariates}

Cognitive function was measured by the Short Portable Mental Status Questionnaire (SPMSQ), which has ten questions dealing with orientation in time and place, personal history, long-term and short-term memory and calculation ability; it has been validated in a Taiwanese population $^{(14,15)}$. With total score ranging from 0 to 10 , correct answers were coded 1, whereas errors were coded 0 . In the present study, participants were grouped into those who did not incur errors (SPMSQ $=10$, intact cognition), those who incurred one or two errors (SPMSQ $=8-9$, mild impairment) and those who incurred three or more errors (SPMSQ $=0-7$, moderate to severe impairment).

Physical function was measured by the physical functioning subscale of the SF-36 Health Survey in a validated traditional Chinese version ${ }^{(16)}$ and was transformed into norm-based scores in which a higher score indicates better physical function ${ }^{(17)}$.

Baseline demographic characteristics, socio-economic status, health and other eating behaviour variables included age, gender, education, marital status, ethnicity, perceived financial status (obtained from the question 'Do you have enough money?'; options for the answer were 'more than enough', 'just enough' and 'not enough'), smoking, alcohol drinking, physical function, chewing ability and nutrition knowledge awareness ('Do you pay attention to nutritional information?; options for the answer were 'always', 'sometimes' and 'rarely'). The number of comorbidities at baseline was the sum of twelve chronic diseases to reflect general health status. Food preparer and shopping frequency ${ }^{(12)}$ were taken into account for social connectedness.

Survival time was the interval between the date of interview and the date of death or 31 December 2008, when censored for survivors. We considered deaths both from baseline and 2 years after the interview. 


\section{Statistical analysis}

Data were weighted to represent the elderly population in Taiwan during 1999-2000. The $\chi^{2}$ test was used for categorical variables across cooking frequency groups. Cox proportional-hazards models were used to assess the association between predictors and survivorship. In multivariable analyses, physical function, cognitive function and nutrition knowledge awareness were included in the models sequentially to elucidate possible mechanisms. Gender-by-cooking frequency interaction terms were estimated to explore gender differences in the cooking-survival relationship. All analyses were performed using the SAS statistical software package version $9 \cdot 1 \cdot 3$ (SAS Institute Inc., Cary, NC, USA) and SUDAAN version $10 \cdot 0$ (RTI International, Research Triangle Park, NC, USA) was used to adjust for the design effect of sampling.

\section{Results}

During the maximal 10-year follow-up, 695 participants died. Tables 1 and 2 present the distributions of demography and behavioural variables across four cooking frequencies. There was an inverse association between cooking frequency and later mortality. Nearly half (43\%) of the study population never cooked, followed by those who cooked 1-2 times/week (17\%), 3-5 times/week (9\%) and $>5$ times/week (31\%). The majority of the study population were aged $<75$ years, males, financially sufficient, non-drinkers of alcohol, non-smokers, engaged in self food preparation, shopped less than once weekly, went out in a private vehicle, were without chewing difficulty, did not eat outside and did not pay attention to nutrition knowledge. About one-quarter had the worst physical function status (physical functioning $<45$ ) and more than half had no detectable cognitive impairment (SPMSQ, 0 error). The highest cooking frequencies ( $>5$ times/week) were associated with younger elders, female gender, less education, unmarried or not living together, non-drinking, non-smoking, greater access to public transportation, cycling or walking and shopping more than once weekly. With regard to BMI, the distributions were significantly different; there were no systematic associations with cooking frequency.

It can be seen that those who reported to cook most frequently prepared food themselves, dined with a spouse but not with others, rarely paid attention to nutrition knowledge and had no chewing difficulty. In terms of food and nutrient intakes, frequent cooking was associated with higher consumption of fat and oil and vegetables, but lower meat intake. The nutrient densities (per $4184 \mathrm{~kJ} /$ $1000 \mathrm{kcal}$ ) of those who cooked most frequently were high in dietary fibre, vitamin $\mathrm{C}$ and $\mathrm{Ca}$, and lower in cholesterol (Table 3).

Table 4 shows the Cox models predicting survival. The crude model shows that cooking 1-2 times/week (hazard ratio $(\mathrm{HR})=0 \cdot 71 ; 95 \% \mathrm{CI}, 0 \cdot 55,0 \cdot 92), 3-5$ times/week

Table 1 Survivorship and demography by cooking frequency: free-living elderly participants aged $\geq 65$ years ( $n$ 1888), Elderly Nutrition and Health Survey in Taiwan, 1999-2000t

\begin{tabular}{|c|c|c|c|c|c|c|}
\hline \multirow[b]{2}{*}{ Descriptor } & \multirow[b]{2}{*}{$n \ddagger$} & \multicolumn{4}{|c|}{ Weekly cooking frequency } & \multirow[b]{2}{*}{$P \S$} \\
\hline & & Never & $1-2$ times & 3-5 times & $>5$ times & \\
\hline Number of participants & 1888 & $43 \cdot 2$ & $17 \cdot 0$ & $8 \cdot 5$ & $31 \cdot 3$ & \\
\hline Survivorship (\%) & 1193 & $54 \cdot 2$ & $63 \cdot 1$ & $68 \cdot 4$ & $74 \cdot 2$ & $<0.001$ \\
\hline Mortality ID per 1000 person-years & & $70 \cdot 5$ & $48 \cdot 9$ & $43 \cdot 5$ & $32 \cdot 7$ & \\
\hline Duration of observation (median, years) & & $8 \cdot 48$ & $8 \cdot 78$ & $8 \cdot 86$ & 8.92 & \\
\hline Gender, men (\%) & 953 & $72 \cdot 2$ & $63 \cdot 6$ & $42 \cdot 7$ & $25 \cdot 0$ & $<0.001$ \\
\hline Age at baseline (\%) & & & & & & $<0.001$ \\
\hline $65-69$ years & 621 & $26 \cdot 2$ & $26 \cdot 1$ & $36 \cdot 1$ & $34 \cdot 9$ & \\
\hline 70-74 years & 654 & $28 \cdot 5$ & $40 \cdot 3$ & $35 \cdot 6$ & $32 \cdot 0$ & \\
\hline 75-97 years & 613 & $45 \cdot 4$ & $33 \cdot 6$ & $28 \cdot 3$ & $33 \cdot 1$ & \\
\hline Ethnicity, indigenous (\%) & 193 & $1 \cdot 1$ & $3 \cdot 6$ & $4 \cdot 4$ & $1 \cdot 6$ & 0.002 \\
\hline Education (\%) & & & & & & $<0.001$ \\
\hline Illiterate & 655 & $34 \cdot 1$ & $31 \cdot 5$ & $35 \cdot 7$ & $42 \cdot 4$ & \\
\hline Elementary school and below & 839 & $42 \cdot 8$ & $34 \cdot 3$ & $36 \cdot 1$ & $39 \cdot 8$ & \\
\hline High school, college and above & 387 & $22 \cdot 6$ & $32 \cdot 8$ & $28 \cdot 1$ & $17 \cdot 3$ & \\
\hline Marital status (\%) & & & & & & $0 \cdot 001$ \\
\hline Never & 45 & $1 \cdot 4$ & $2 \cdot 7$ & $2 \cdot 4$ & $4 \cdot 2$ & \\
\hline Married and live together & 1247 & $72 \cdot 5$ & $65 \cdot 8$ & $66 \cdot 9$ & $56 \cdot 9$ & \\
\hline Separated, divorced or widowed & 594 & $25 \cdot 9$ & $31 \cdot 6$ & $30 \cdot 1$ & $39 \cdot 0$ & \\
\hline Perceived financial status (\%) & & & & & & 0.070 \\
\hline Enough or just enough & 1275 & $73 \cdot 5$ & $81 \cdot 0$ & $72 \cdot 4$ & $69 \cdot 9$ & \\
\hline Some difficulty & 451 & $18 \cdot 9$ & $14 \cdot 3$ & $20 \cdot 9$ & $21 \cdot 1$ & \\
\hline Very difficult & 101 & $2 \cdot 9$ & $2 \cdot 4$ & $5 \cdot 3$ & $6 \cdot 7$ & \\
\hline
\end{tabular}

ID, incidence density.

tPercentages are weighted to reflect their representation in the population.

$\ddagger$ Total sample size is 1888; cases with missing values were not included for the relevant variable.

§From $\chi^{2}$ test (SUDAAN version 10·0; RTI International). 
Table 2 Behavioural characteristics and health status by cooking frequency: free-living elderly participants aged $\geq 65$ years $(n 1888$ ), Elderly Nutrition and Health Survey in Taiwan, 1999-2000†

\begin{tabular}{|c|c|c|c|c|c|c|}
\hline \multirow[b]{2}{*}{ Descriptor } & \multirow[b]{2}{*}{$n \ddagger$} & \multicolumn{4}{|c|}{ Weekly cooking frequency } & \multirow[b]{2}{*}{$P \S$} \\
\hline & & Never & $1-2$ times & 3-5 times & $>5$ times & \\
\hline Alcohol drinker (\%) & & & & & & $<0.001$ \\
\hline Never & 1386 & $69 \cdot 7$ & $65 \cdot 6$ & $77 \cdot 0$ & $85 \cdot 9$ & \\
\hline Former & 126 & $9 \cdot 7$ & $8 \cdot 2$ & 4.5 & $2 \cdot 4$ & \\
\hline Current & 372 & $20 \cdot 6$ & $25 \cdot 5$ & $17 \cdot 6$ & $11 \cdot 4$ & \\
\hline Smoker (\%) & & & & & & $<0.001$ \\
\hline Never & 1226 & $49 \cdot 3$ & $59 \cdot 1$ & $71 \cdot 7$ & $79 \cdot 6$ & \\
\hline Former & 239 & $20 \cdot 9$ & $16 \cdot 1$ & $9 \cdot 4$ & $8 \cdot 5$ & \\
\hline Current & 414 & $29 \cdot 6$ & $24 \cdot 8$ & $18 \cdot 0$ & $11 \cdot 6$ & \\
\hline Physical functioning score (\%) & & & & & & $<0.001$ \\
\hline$<45$ & 480 & $32 \cdot 1$ & $19 \cdot 5$ & $17 \cdot 6$ & $21 \cdot 2$ & \\
\hline $45-53 \cdot 9$ & 596 & $26 \cdot 5$ & $31 \cdot 0$ & $30 \cdot 5$ & $37 \cdot 8$ & \\
\hline $54-57 \cdot 9$ & 507 & $22 \cdot 7$ & $28 \cdot 6$ & $33 \cdot 9$ & $26 \cdot 7$ & \\
\hline$\geq 58$ & 274 & $15 \cdot 7$ & $20 \cdot 3$ & $17 \cdot 2$ & $14 \cdot 4$ & \\
\hline SPMSQ (\%) & & & & & & $<0.001$ \\
\hline 0 errors & 974 & $52 \cdot 6$ & $64 \cdot 9$ & $59 \cdot 7$ & $53 \cdot 5$ & \\
\hline $1-2$ errors & 538 & $25 \cdot 4$ & $21 \cdot 6$ & $25 \cdot 9$ & $30 \cdot 3$ & \\
\hline$\geq 3$ errors & 357 & $20 \cdot 5$ & $13 \cdot 5$ & $13 \cdot 1$ & $16 \cdot 2$ & \\
\hline Transportation (\%) & & & & & & $<0.001$ \\
\hline Never go out & 149 & $16 \cdot 1$ & $4 \cdot 8$ & $2 \cdot 9$ & $4 \cdot 3$ & \\
\hline Private vehicle (motorcycle or car) & 911 & $52 \cdot 0$ & $48 \cdot 2$ & $53 \cdot 2$ & $37 \cdot 4$ & \\
\hline Public transportation & 429 & $12 \cdot 6$ & $24 \cdot 0$ & $19 \cdot 3$ & $26 \cdot 2$ & \\
\hline Bicycle & 167 & $11 \cdot 6$ & $8 \cdot 4$ & $8 \cdot 2$ & $13 \cdot 4$ & \\
\hline Walking & 231 & $7 \cdot 8$ & $14 \cdot 6$ & $16 \cdot 4$ & $18 \cdot 7$ & \\
\hline Weekly shopping frequency (\%) & & & & & & $<0.001$ \\
\hline Less than once & 923 & $61 \cdot 7$ & $43 \cdot 4$ & $28 \cdot 4$ & $33 \cdot 3$ & \\
\hline Once & 264 & $9 \cdot 2$ & $15 \cdot 5$ & $19 \cdot 0$ & $15 \cdot 0$ & \\
\hline 2-4 times & 391 & $15 \cdot 1$ & $24 \cdot 4$ & $32 \cdot 5$ & $27 \cdot 2$ & \\
\hline Every day & 278 & $11 \cdot 7$ & $15 \cdot 0$ & $15 \cdot 3$ & $23 \cdot 8$ & \\
\hline BMI (\%) & & & & & & $<0.001$ \\
\hline$<18.5 \mathrm{~kg} / \mathrm{m}^{2}$ & 101 & $5 \cdot 6$ & $3 \cdot 8$ & $2 \cdot 4$ & $6 \cdot 9$ & \\
\hline $18 \cdot 5-23 \cdot 9 \mathrm{~kg} / \mathrm{m}^{2}$ & 646 & $38 \cdot 0$ & $35 \cdot 5$ & $39 \cdot 8$ & $34 \cdot 6$ & \\
\hline $24 \cdot 0-26 \cdot 9 \mathrm{~kg} / \mathrm{m}^{2}$ & 416 & $17 \cdot 8$ & $26 \cdot 2$ & $19 \cdot 7$ & $24 \cdot 5$ & \\
\hline$\geq 27 \cdot 0 \mathrm{~kg} / \mathrm{m}^{2}$ & 255 & $10 \cdot 8$ & $11 \cdot 3$ & $19 \cdot 6$ & $13 \cdot 6$ & \\
\hline
\end{tabular}

SPMSQ, Short Portable Mental Status Questionnaire.

tPercentages are weighted to reflect their representation in the population.

$\ddagger$ Total sample size is 1888; cases with missing values were not included for the relevant variable.

§From $\chi^{2}$ test (SUDAAN version 10.0; RTI International).

$(\mathrm{HR}=0 \cdot 59 ; 95 \% \mathrm{CI}, 0 \cdot 43,0 \cdot 80)$ and $>5$ times/week $(\mathrm{HR}=0 \cdot 47 ; 95 \% \mathrm{CI}, 0 \cdot 36,0 \cdot 61)$ was associated with a significantly lower mortality risk compared with no cooking activity. Likewise, Model 1 adjusted for covariates (gender, age, education, ethnicity, alcohol drinking, smoking, region, financial status, number of co-morbidities, marital status, dinner companions, shopping and chewing ability) showed that people who cooked more than never were at a lower risk of mortality than those who did not. The lowest risk of mortality was for cooking $>5$ times/week $(\mathrm{HR}=0 \cdot 56 ; 95 \%$ CI, 0·39, 0·81).

Further control for physical function and cognitive function (Model 2) and nutrition knowledge (Model 3) did not alter the estimates substantially. In Model 4, although the relationship was attenuated with the exclusion of those who died in the first 2 years, those cooking most frequently still had significantly lowest risk of death $(\mathrm{HR}=0 \cdot 65 ; 95 \%$ CI, 0•44, 0.95). In a sub-data analysis which included only the participants capable of cooking activity without health difficulty (Model 5), those cooking $>5$ times/week retained the lowest risk of death $(\mathrm{HR}=0 \cdot 61 ; P$ for trend $=0 \cdot 008)$.
For single household elders (Model 6), although not significant, most frequent cooking still had the lowest risk of mortality $(\mathrm{HR}=0 \cdot 60 ; P$ for trend $=0 \cdot 230)$.

A gender-by-cooking frequency joint effect on survival was evident in a model with a significant interaction $(P=0.01)$ based on Model 3 (Fig. 1). The differences in risk of death were $24 \%$ for men and $51 \%$ for women when comparison was made between those who cooked $>5$ times/week and those who never cooked.

\section{Discussion}

\section{Cooking and survivorship}

We explored whether food preparation could predict long-term survival in an elderly population and whether the effect would remain when functional limitations were considered. Modelling with different sets of covariates and with subset data demonstrated the robustness of the finding that highly frequent cooking activity predicts survival among elderly Taiwanese. However, this desirable effect of 
Table 3 Food and nutritional characteristics and health status by cooking frequency: free-living elderly participants aged $\geq 65$ years ( $n$ 1888), Elderly Nutrition and Health Survey in Taiwan, 1999-2000t

\begin{tabular}{|c|c|c|c|c|c|c|}
\hline \multirow[b]{2}{*}{ Descriptor } & \multirow[b]{2}{*}{$n \ddagger$} & \multicolumn{4}{|c|}{ Weekly cooking frequency } & \multirow[b]{2}{*}{$P \S$} \\
\hline & & Never & $1-2$ times & $3-5$ times & $>5$ times & \\
\hline Food preparer (\%) & & & & & & $<0.001$ \\
\hline No self-cooked food & 115 & $15 \cdot 1$ & $5 \cdot 2$ & $1 \cdot 6$ & $0 \cdot 2$ & \\
\hline Self & 752 & $8 \cdot 6$ & $24 \cdot 1$ & $52 \cdot 1$ & $84 \cdot \overline{6}$ & \\
\hline Spouse & 475 & $33 \cdot 8$ & $34 \cdot 3$ & $21 \cdot 4$ & $8 \cdot 1$ & \\
\hline Family, friends, neighbours or housemaid & 532 & $42 \cdot 5$ & $36 \cdot 4$ & $24 \cdot 9$ & $7 \cdot 1$ & \\
\hline Dining companions (\%) & & & & & & $<0.001$ \\
\hline None & 330 & $11 \cdot 5$ & $7 \cdot 57$ & $11 \cdot 8$ & $30 \cdot 2$ & \\
\hline Spouse & 717 & $34 \cdot 6$ & $35 \cdot 0$ & $38 \cdot 8$ & $36 \cdot 9$ & \\
\hline Family, friend or neighbours & 837 & $53 \cdot 7$ & $57 \cdot 4$ & $49 \cdot 4$ & $32 \cdot 5$ & \\
\hline Eating out (\%) & & & & & & 0.340 \\
\hline Yes & 89 & $5 \cdot 3$ & $8 \cdot 3$ & $6 \cdot 3$ & $4 \cdot 8$ & \\
\hline No & 1799 & $94 \cdot 7$ & $91 \cdot 7$ & $93 \cdot 7$ & $95 \cdot 2$ & \\
\hline Nutrition knowledge awareness (\%) & & & & & & $<0.001$ \\
\hline Yes & 687 & $31 \cdot 1$ & $43 \cdot 2$ & $53 \cdot 7$ & $35 \cdot 1$ & \\
\hline No & 1201 & $68 \cdot 9$ & $56 \cdot \overline{8}$ & $46 \cdot 3$ & $64 \cdot 9$ & \\
\hline Chewing difficulty (\%) & & & & & & 0.002 \\
\hline Yes & 705 & $39 \cdot 5$ & $33 \cdot 7$ & $32 \cdot 9$ & $32 \cdot 6$ & \\
\hline No & 1144 & $55 \cdot 8$ & $65 \cdot 7$ & $65 \cdot 8$ & $66 \cdot 7$ & \\
\hline \multicolumn{7}{|l|}{ Mean daily food intakes per $4184 \mathrm{~kJ}(1000 \mathrm{kcal}) \|$} \\
\hline Rice and grains $(\mathrm{g})$ & & 147 & 152 & 136 & 149 & $0 \cdot 103$ \\
\hline Fat and oil (g) & & $8 \cdot 2$ & $8 \cdot 0$ & $9 \cdot 4$ & $10 \cdot 5$ & $<0.001$ \\
\hline Meat $(\mathrm{g})$ & & $49 \cdot \overline{5}$ & $46 \cdot 8$ & $49 \cdot 0$ & $39 \cdot 2$ & 0.030 \\
\hline Fish and shellfish (g) & & $46 \cdot 0$ & $39 \cdot 4$ & $33 \cdot 5$ & $40 \cdot 0$ & 0.062 \\
\hline Eggs $(\mathrm{g})$ & & $11 \cdot 1$ & $11 \cdot 4$ & $8 \cdot 4$ & $8 \cdot 8$ & $0 \cdot 196$ \\
\hline Dairy $(\mathrm{g})$ & & $34 \cdot 7$ & $28 \cdot 1$ & $26 \cdot 1$ & $29 \cdot 3$ & 0.441 \\
\hline Soyabean products $(\mathrm{g})$ & & $27 \cdot 5$ & $29 \cdot 6$ & $27 \cdot 0$ & $24 \cdot 6$ & $0 \cdot 808$ \\
\hline Vegetables $(\mathrm{g})$ & & 204 & 210 & 191 & 245 & 0.008 \\
\hline Fruit $(\mathrm{g})$ & & 94 & 102 & 111 & 117 & 0.132 \\
\hline \multicolumn{7}{|l|}{$\begin{array}{l}\text { Mean daily energy intake and nutrient densities } \\
\text { per } 4184 \mathrm{~kJ}(1000 \mathrm{kcal})\end{array}$} \\
\hline Energy $(\mathrm{kJ})$ & & 6866 & 7795 & 6887 & 6682 & 0.005 \\
\hline Energy (kcal) & & 1641 & 1863 & 1646 & 1597 & 0.005 \\
\hline Protein $(\mathrm{g})$ & & $43 \cdot 2$ & $42 \cdot 2$ & $43 \cdot 0$ & $41 \cdot 5$ & 0.361 \\
\hline Fat $(\mathrm{g})$ & & $29 \cdot \overline{8}$ & $29 \cdot \overline{5}$ & $27 \cdot 8$ & $28 \cdot 3$ & 0.301 \\
\hline Cholesterol (mg) & & 142 & 135 & 123 & 116 & 0.005 \\
\hline Carbohydrate (g) & & 139 & 140 & 145 & 145 & 0.075 \\
\hline Dietary fibre (g) & & $11 \cdot 6$ & $12 \cdot 4$ & $12 \cdot 2$ & $14 \cdot 0$ & 0.002 \\
\hline Vitamin A (IU) & & 6354 & 5232 & 5949 & 7338 & 0.067 \\
\hline Vitamin $\mathrm{E}(\alpha-\mathrm{TE}, \mathrm{mg})$ & & $4 \cdot 68$ & $5 \cdot 13$ & $5 \cdot 38$ & $5 \cdot 24$ & 0.060 \\
\hline Vitamin $B_{1}(\mathrm{mg})$ & & 0.67 & 0.68 & $0 \cdot 82$ & 0.69 & 0.465 \\
\hline Vitamin $B_{2}(\mathrm{mg})$ & & 0.83 & 0.88 & 0.99 & 0.91 & 0.266 \\
\hline Vitamin $\mathrm{B}_{6}(\mathrm{mg})$ & & $0 \cdot 72$ & $0 \cdot 70$ & $0 \cdot 74$ & 0.72 & 0.857 \\
\hline Vitamin C (mg) & & 89 & 100 & 87 & 118 & $<0.001$ \\
\hline $\mathrm{Ca}(\mathrm{mg})$ & & 379 & 414 & 443 & 442 & 0.001 \\
\hline $\mathrm{Fe}(\mathrm{mg})$ & & $7 \cdot 34$ & $7 \cdot 56$ & $7 \cdot 36$ & $7 \cdot 80$ & 0.618 \\
\hline $\mathrm{Na}(\mathrm{mg})$ & & 3106 & 2894 & 2981 & 3183 & 0.129 \\
\hline $\mathrm{Mg}(\mathrm{mg})$ & & 144 & 149 & 147 & 152 & 0.255 \\
\hline $\mathrm{K}(\mathrm{mg})$ & & 1463 & 1530 & 1429 & 1556 & 0.257 \\
\hline
\end{tabular}

$\alpha$-TE, $\alpha$-tocopherol equivalents.

tPercentages are weighted to reflect their representation in the population.

$\ddagger$ Total sample size is 1888 ; cases with missing values were not included for the relevant variable.

SFrom $\chi^{2}$ test (SUDAAN version 10·0; RTI International).

॥From a $24 \mathrm{~h}$ dietary recall.

cooking activity is likely to benefit from physical and/or cognitive functions and from nutrition knowledge, in addition to other factors, which were evaluated in the present study.

We know that dietary diversity is associated with greater survival in this cohort ${ }^{(18)}$. Along these lines, we have now found that those who cook more frequently have a better diet and more favourable nutrient densities. It is therefore possible that cooking relates to survivorship through food choice and quality; the reverse may also apply. To some extent, this relationship may also be due to a link between shopping ${ }^{(12)}$ and cooking frequencies.

The characteristics of those who cook more often are that they are women who live and eat alone, have less tertiary education (post secondary technical or university), have less substance use (alcohol or tobacco), have better physical function and walk more, and engage in frequent shopping. The gender difference might result 
Table 4 Hazard ratios (HR) and $95 \%$ confidence intervals for cooking frequency and risk of deatht: free-living elderly participants aged $\geq 65$ years $(n 1888)$, Elderly Nutrition and Health Survey in Taiwan, 1999-2000

\begin{tabular}{|c|c|c|c|c|c|c|c|}
\hline & \multicolumn{6}{|c|}{ Weekly cooking frequency } & \multirow[b]{3}{*}{$P$ for trenc } \\
\hline & \multicolumn{2}{|c|}{$1-2$ times } & \multicolumn{2}{|c|}{ 3-5 times } & \multicolumn{2}{|c|}{$>5$ times } & \\
\hline & HR & $95 \% \mathrm{Cl}$ & $\mathrm{HR}$ & $95 \% \mathrm{Cl}$ & $\mathrm{HR}$ & $95 \% \mathrm{Cl}$ & \\
\hline Crude & $0 \cdot 71^{*}$ & $0.55,0.92$ & $0.59^{\star *}$ & $0.43,0.80$ & $0.47^{\star \star \star}$ & $0.36,0.61$ & $<0.001$ \\
\hline Model $1 \ddagger$ & 0.95 & $0.74,1.22$ & 0.74 & $0.52,1.05$ & $0 \cdot 56^{\star \star}$ & $0.39,0.81$ & 0.004 \\
\hline Model $2 \S$ & $1 \cdot 01$ & $0.79,1.30$ & 0.78 & $0.54,1.12$ & $0.59^{\star \star}$ & $0.41,0.86$ & 0.009 \\
\hline Model 3॥ & $1 \cdot 01$ & $0.79,1.29$ & 0.80 & $0.56,1 \cdot 13$ & $0.59^{\star \star}$ & $0.41,0.86$ & 0.009 \\
\hline Model 4 & 1.07 & $0.79,1.45$ & 0.90 & $0.65,1 \cdot 24$ & $0 \cdot 65^{\star}$ & $0.44,0.95$ & 0.030 \\
\hline Model 5tt & $1 \cdot 06$ & $0.81,1.37$ & 0.83 & $0.59,1.17$ & $0.61^{\text {** }}$ & $0.43,0.86$ & 0.008 \\
\hline Model 6ł & 0.95 & $0 \cdot 32,2 \cdot 83$ & $0 \cdot 81$ & $0 \cdot 26,2 \cdot 51$ & 0.60 & $0.27,1.34$ & 0.230 \\
\hline
\end{tabular}

HR was significant: ${ }^{\star} P<0.05,{ }^{* *} P<0.01,{ }^{\star * \star} P<0.001$.

tHR estimated by Cox proportional-hazards models, with 'Never cook' as the reference group.

¥Model 1: adjusted for gender, age (continuous), education, ethnicity, drinking alcohol, smoking, region, financial status, number of co-morbidities, marital status, dining companions, shopping and chewing ability.

$\S$ Model 2: adjusted for Model 1 covariates plus physical function and cognitive function.

IIModel 3: adjusted for Model 2 covariates plus nutrition knowledge.

- Model 4: Model 3 for a sub-sample in which those who died during the first 2 years were excluded $(n 1775)$.

t+Model 5: Model 3 for a sub-sample in which those who were unable to cook due to difficulty with physical function were excluded $(n 1809)$. ¥¥Model 6: Model 3 for a sub-sample which only included those who were single household elders $(n 330)$.

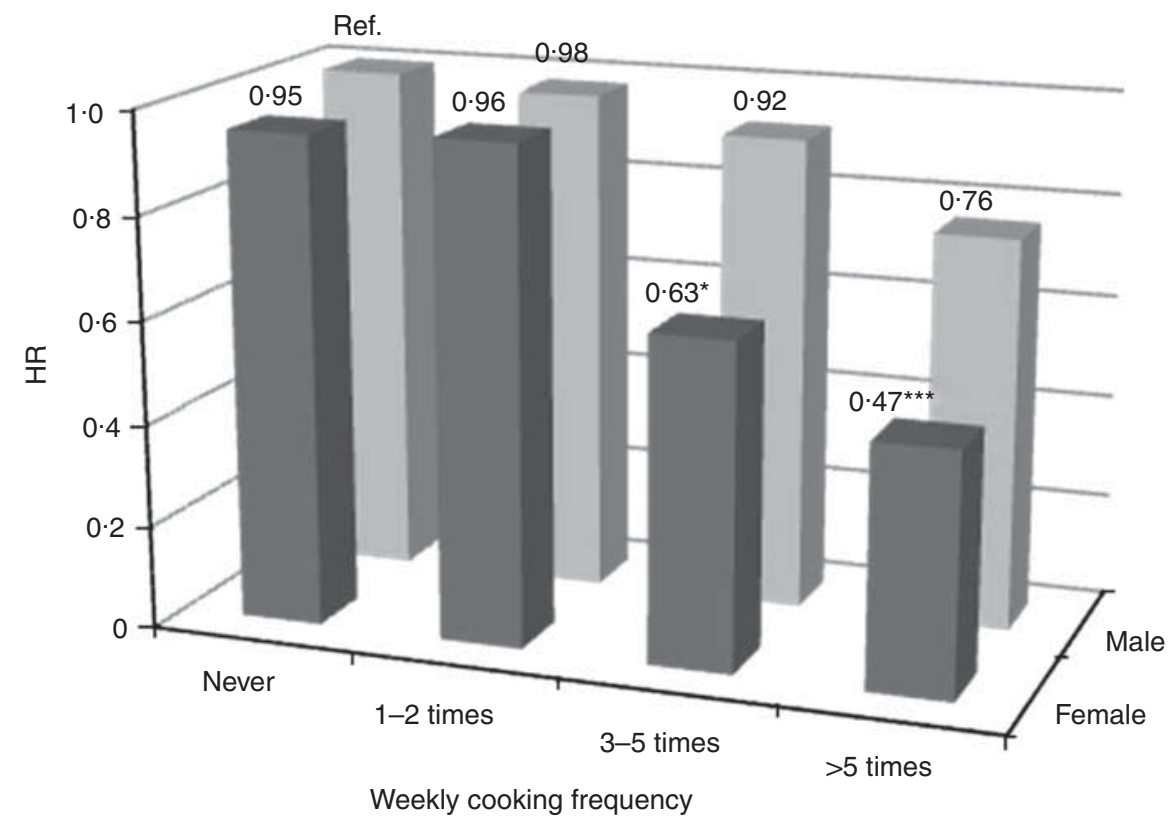

Fig. 1 Hazard ratios (HR) for eight gender-cooking categories from the model adjusted by gender, age, education, ethnicity, alcohol drinking, smoking, region, financial status, number of co-morbidities, marital status, dining companions, shopping and chewing ability, physical function, cognitive function and nutrition knowledge: free-living elderly participants aged $\geq 65$ years ( $n$ 1888), Elderly Nutrition and Health Survey in Taiwan, 1999-2000. HR was significant: ${ }^{\star} P<0 \cdot 05,{ }^{\star \star \star} P<0 \cdot 001$. Interaction of cooking frequency and gender was significant, $P=0.01$ (Ref., reference category)

from cultural stereotypes with regard to domestic roles like cooking, but, all the same, is an important risk factor for mortality among the principally Han Chinese in the present study. This difference has implications for men who do not cook so often, at least in Taiwan. The risk to men is underscored by a Swiss study which showed that men were, with doubtful justification, more positive about the nutritional value and taste of convenience foods compared with women ${ }^{(19)}$. Nevertheless, women who cook have better food choices and nutrient densities than do men (data not shown). Since indigenous Taiwanese are also included in the present nationally representative survey, we remain cautious about any peculiar attribution of risk with regard to ethnicity.

The findings for level of education may relate to socioeconomic differences where alternatives to domestic food preparation and cooking are more available for the tertiary educated. Notwithstanding this difference in cooking-related mortality by education, when adjustment is made for it, cooking frequency still matters. On the 
question of substance use (alcohol and tobacco), it would seem that these behaviours are associated with less cooking. It is therefore a possibility that they may exert their known adverse effects on survival, in part, by way of food preparation and cooking, or vice versa. It is interesting to consider whether active interest in food may decrease substance use. However, these several variables were all obtained at the same baseline and therefore questions of cause and effect can only be conjectural.

It is not surprising that the older aged cook less often than their younger counterparts, which may simply relate to increasing frailty and less independence. If such factors associated with age were mitigated, then cooking frequency might be maintained and longevity improved in a multifactorial fashion. In that case cooking frequency might prolong life by social pathways. Nevertheless, health outcomes are more favourable where there is a sense of control, independence and autonomy as emphasised in the WHO report on the social determinants of disease ${ }^{(20)}$ and cooking may contribute to this kind of health advantage.

We also found a relationship between a sense of nutrition knowledge and cooking frequency. Again, cooking behaviour which takes account of health may, in part, represent the psychosocial determinants of health ${ }^{(21)}$.

\section{Linking cooking as an activity witb bealtb impairment and survival}

As indicated, there may be several pathways which link cooking to survival, if it is presumed that there may be a pathogenetic connection. These might include knowledge and skill, health and nutrition literacy, and health-seeking behaviour $^{(21)}$. Health status is also likely to be important.

There will be incentives and disincentives to cooking which change with age. Some will relate to taste and smell which decline with age ${ }^{(22)}$. The quest for salt may reflect these declines in taste perception ${ }^{(23)}$. Chewing, which represents a number of functional changes with age, is also an important predictor of food intake and survival and we adjusted for it with retention of the cooking-survival linkage ${ }^{(24)}$. In short, there are biological changes in food preference which are likely to influence the inclination to cook. For these reasons we have considered the link between cooking and survival not only from the beginning of the observation period in 1999-2000, but also with a 2-year delay. The findings were similar.

\section{Limitations of the present study}

Data were measured only once at baseline. However we have appraised the analysis excluding people with physical difficulty to cook (those with severe cognitive impairment were already excluded; see below) and the results remained similar. In addition, we retained in our analysis those who had help with cooking so that the apparent benefit may be achieved where there is disability by acknowledging the need to cook and securing assistance. Although our study has not provided intermediate health outcomes between cooking behaviour and mortality, we consider that the companion health behaviours and status at baseline are likely to have been persistent and contributory or otherwise to the ultimate mortality outcome.

Our sample was drawn from the community of free-living elderly and information was obtained at the household level. It does not, therefore, include institutionalized elders. In the case of those with severe cognitive impairment who remained at home, they were also not included, and this has been acknowledged in our exclusion criteria. Because of limited cooking ability and safety, they might have influenced our findings. From our modelling, we find that those with functional limitations affecting the ability to cook contributed disproportionately to low frequency-ofcooking-related mortality. Nevertheless, with comprehensive adjustments, the remainder of the population remained at risk of reduced survival on account of low frequency of cooking. Whether a household is single member or comprises several individuals, cooking frequency is of benefit to survival.

Cooking as a surrogate for other phenomena, for health status or socio-economic circumstance, is possible. Our modelling has been extensive and endeavoured to cover these possible explanatory variables, although there may yet be residual confounding present. Even so, cooking is an integrated activity by its nature, embracing a range of psychosocial (such as mood and demography) and biomedical phenomena (such as sensory inputs and disability) which stands in its own right, so minimizing the problem of residual confounding.

\section{Conclusions}

Elders who cook with moderate frequency have a lower risk of mortality. This is probably related to both better food choices and health. The present findings encourage public health policy which emphasizes the development and maintenance of cooking skills, interest and performance as a way to extend healthy lives.

\section{Acknowledgements}

The study was sponsored by the Department of Health (DOH; grant number DOH098TD-F-113-098029) and the National Health Research Institutes (NHRI), Taiwan. No author has any conflict of interest with regard to this paper. R.C.-Y.C. and M.L.W. conceived of and executed the project. R.C.-Y.C. performed the statistical analyses with supervision by M.-S.L. The paper was drafted by R.C.-Y.C. in consultation with M.L.W. M.-S.L., M.L.W. and Y.-H.C. interpreted and revised the paper. The data set was developed by Dr Wen-Harn Pan and Su-Hao Tu at Academia Sinica. 
The data management units at Academia Sinica, NHRI and $\mathrm{DOH}$ are thanked for their contribution to data protection and confidentiality protocols.

\section{References}

1. Rowe JW \& Kahn RL (1997) Successful ageing. Gerontologist 37, 433-440.

2. Strawbridge WJ, Wallhagen MI \& Cohen RD (2002) Successful ageing and well-being: self-rated compared with Rowe and Kahn. Gerontologist 42, 727-733.

3. World Health Organization (2011) Healthy ageing is vital for development. http://www.who.int/mediacentre/news/ releases/release24/en/ (accessed August 2011).

4. Zanjani FA, Schaie KW \& Willis SL (2003) Predicting mortality using eight health behaviors domains. Gerontologist 43, 333-334.

5. Chenhall C (2010) Improving cooking and food preparation skills: a synthesis of the evidence to inform program and policy. http://www.hc-sc.gc.ca/fn-an/nutrition/child-enfant/ cfps-acc-synthes-eng.php (accessed October 2011).

6. Wahlqvist ML (2009) Connected Community and Household Food Based Strategy (CCH-FBS): its importance for health, food safety, sustainability and security in diverse localities. Ecol Food Nutr 48, 457-481.

7. Wahlqvist M (2011) Nutrition in adulthood. In Food and Nutrition: Food and Health Systems in Australia and New Zealand, pp. 469-472. Crows Nest, NSW: Allen \& Unwin.

8. Wahlqvist M (2002) Asian migration to Australia: food and health consequences. Asia Pac J Clin Nutr 11, Suppl. 3, S562-S568

9. Park SM, Jang SN \& Kim DH (2010) Gender differences as factors in successful ageing: a focus on socioeconomic status. J Biosoc Sci 42, 99-111.

10. Wang YJ (2009) The phenomenon of Fu, Pei-Mei: the economy and community change in Taiwan 1960s. Master Thesis, Feng Chia University.

11. Stewart H, Blisard N \& Jolliffe D (2006) Let's Eat Out Americans Weigh Taste, Convenience, and Nutrition. Economic Information Bulletin no. EIB-19. Washington, DC: USDA, Economic Research Service.
12. Chang YH, Chen RCY, Wahlqvist ML et al. (2011) Frequent shopping by men and women increases survival in the older Taiwanese population. J Epidemiol Community Health (Epublication ahead of print version).

13. Pan WH, Hung YT, Shaw SS et al. (2005) Elderly nutrition and health survey in Taiwan (1999-2000): research design, methodology and content. Asia Pac J Clin Nutr 14, 203-210.

14. Pfeiffer E (1975) A short portable mental status questionnaire for the assessment of organic brain deficit in elderly patients. I Am Geriatr Soc 23, 433-441.

15. Hsiao SH, Chiu HC \& Liu HW (1994) A replication of multidimensionality of Activities of Daily Living (ADL): on the elderly in Southern Taiwan. Kaohsiung J Med Sci 10, 449-457 (in Chinese).

16. Lu JF, Tseng HM \& Tsai YJ (2003) Assessment of healthrelated quality of life in Taiwan (I): development and psychometric testing of SF-36 Taiwan version. Taiwan J Public Health 22, 501-511 (in Chinese).

17. Ware JE, Kosinski M \& Dewey JE (2000) How to Score Version 2 of the SF-36 ${ }^{\circledR}$ Health Survey, pp. 27-48. Lincoln, RI: QualityMetric Inc.

18. Lee MS, Huang YC \& Su HH (2011) A simple food quality index predicts mortality in elderly Taiwanese. J Nutr Health Aging 15, 815-821.

19. Van Der Horst K, Brunner TA \& Siegrist M (2010) Readymeal consumption: associations with weight status and cooking skills. Public Health Nutr 14, 239-245.

20. Marmot M, Friel S \& Bell R (2008) Closing the gap in a generation: health equity through action on the social determinants of health. Lancet 372, 1661-1669.

21. Mead E, Gittelsohn J \& Roache C (2010) Healthy food intentions and higher socioeconomic status are associated with healthier food choices in an Inuit population. J Hum Nutr Diet 23, 83-91.

22. Epstein F \& Schiffman S (1983) Taste and smell in disease. $N$ Engl J Med 308, 1275-1279, 1337-1343.

23. Kaneda H, Maeshima K \& Goto N (2000) Decline in taste and odor discrimination abilities with age, and relationship between gustation and olfaction. Chem Senses 25, 331-337.

24. Lee MS, Huang YC \& Wahlqvist M (2010) Chewing ability in conjunction with food intake and energy status in later life affects survival in Taiwanese with the metabolic syndrome. J Am Geriatr Soc 58, 1072-1080. 